\title{
Meiotic Chromosome Synapsis-Promoting Proteins Antagonize the Anti-Crossover Activity of Sgs1
}

\author{
Lea Jessop ${ }^{1}$, Beth Rockmill ${ }^{2}$, G. Shirleen Roeder ${ }^{2,3,4}$, Michael Lichten ${ }^{1 *}$ \\ 1 Center for Cancer Research, National Cancer Institute, Bethesda, Maryland, United States of America, 2 Department of Molecular, Cellular, and Developmental Biology, Yale \\ University, New Haven, Connecticut, United States of America, 3 Department of Genetics, Yale University, New Haven, Connecticut, United States of America, 4 Howard \\ Hughes Medical Institute, Yale University, New Haven, Connecticut, United States of America
}

Sgs1, the budding yeast homolog of the mammalian BLM helicase, has been implicated in preventing excess recombination during both vegetative growth and meiosis. Most meiotic crossover (CO) recombination requires full function of a set of yeast proteins (Zip1, Zip2, Zip3, Zip4/Spo22, Mer3, Msh4, and Msh5, termed the SIC or ZMM proteins) that are also required for homologous chromosome synapsis. We report here genetic and molecular assays showing that sgs 1 single mutants display relatively modest increases in CO recombination (less than 1.6-fold relative to wild-type). In contrast, a much greater $\mathrm{CO}$ increase is seen when an sgs 1 mutation is introduced into the $\mathrm{CO}$ - and synapsis-deficient zip1, zip2, zip3, mer3, or msh4 mutants (2- to 8-fold increase). Furthermore, close juxtaposition of the axes of homologous chromosomes is restored. CO restoration in the mutants is not accompanied by significant changes in noncrossover (NCO) recombinant frequencies. These findings show that Sgs 1 has potent meiotic anti-CO activity, which is normally antagonized by SIC/ZMM proteins. Our data reinforce previous proposals for an early separation of meiotic processes that form CO and NCO recombinants.

Citation: Jessop L, Rockmill B, Roeder GS, Lichten M (2006) Meiotic chromosome synapsis-promoting proteins antagonize the anti-crossover activity of Sgs1. PLoS Genet 2(9): e155. DOI: 10.1371/journal.pgen.0020155

\section{Introduction}

DNA double-strand breaks (DSBs) pose a significant risk to cells. Failure to repair DSBs can result in death, while imprecise repair can form translocations, deletions, and other chromosome rearrangements. DSBs are repaired by two distinct mechanisms: end-joining, in which the ends of breaks are ligated, often imprecisely, and homologous recombination, in which breaks are repaired using homologous sequences as a template to form recombinants that are either crossover $(\mathrm{CO})$ or noncrossover $(\mathrm{NCO})$ with regard to flanking parental sequences. Although repair by homologous recombination is generally considered nonmutagenic (but see [1]), the CO outcome has the potential for deleterious genome rearrangement, loss of heterozygosity, or both. Perhaps as a consequence, the rare interhomolog recombination events that do occur during the mitotic cell cycle are infrequently accompanied by crossing over [2].

In contrast, COs are frequent in meiosis, with at least one per homolog pair [3]. COs are an integral part of the interhomolog connections that are necessary for homolog alignment and spindle assembly at metaphase I [4,5]. As a consequence, mutants with either general meiotic recombination defects or specific defects in meiotic COs undergo frequent homolog mis-segregation and gamete death. Even a single pair of chromosomes that fails to cross over is at increased risk of nondisjunction at meiosis I [6-8]. In most organisms where these events have been examined, the total number of interhomolog recombination events is considerably greater than the number of COs [9], and both COs and NCOs are needed to facilitate meiotic homolog pairing $[10,11]$.

The molecular mechanism of meiotic recombination and the factors that determine whether events will produce NCO or $\mathrm{CO}$ products have been studied most extensively in the budding yeast Saccharomyces cerevisiae. Studies in this organism show that meiotic recombination is initiated by DSBs, formed by the meiosis-specific endonuclease Spo11 [12]. Breaks are subsequently resected to generate single-stranded DNA tails with free $3^{\prime}$ ends [13]. Most COs are produced via formation of a semi-stable single end invasion intermediate in which one DSB end interacts with the homolog [14], followed by capture of the second DSB end to form a double Holliday junction (dHJ) intermediate [14-17]. By contrast, most NCOs form via processes that do not appear to involve stable $\mathrm{dHJ}$ intermediates [16,17] and a synthesis-dependent strandannealing mechanism has been suggested [2,16,18].

Evidence for mechanistic separation of $\mathrm{CO}$ and $\mathrm{NCO}$ recombination comes from molecular studies of two classes of mutants that block CO formation without reducing NCOs. Cells lacking the Ndt80 transcription factor or the Cdc5

Editor: James E. Haber, Brandeis University, United States of America

Received April 5, 2006; Accepted August 2, 2006; Published September 22, 2006 A previous version of this article appeared as an Early Online Release on August 2, 2006 (DOI: 10.1371/journal.pgen.0020155.eor).

DOI: 10.1371/journal.pgen.0020155

This is an open-access article distributed under the terms of the Creative Commons Public Domain declaration which stipulates that, once placed in the public domain, this work may be freely reproduced, distributed, transmitted, modified, built upon, or otherwise used by anyone for any lawful purpose.

Abbreviations: $\mathrm{AA}$, axial association; $\mathrm{CO}$, crossover; $\mathrm{dHJ}$, double Holliday junction; DSB, double-strand break; NCO, noncrossover; SC, synaptonemal complex; SIC synapsis initiation complex; ZMM, Zip Msh Mer proteins

* To whom correspondence should be addressed. E-mail: lichten@helix.nih.gov 


\section{Synopsis}

Most eukaryotic cells are diploid (two copies of each chromosome per cell), but gametes (in animals, sperm and eggs) are haploid (one chromosome copy). Gametes are produced from diploid cells during meiosis. The two copies of each chromosome are brought together in end-to-end alignment (synapsis), and then are connected by crossover recombination, which involves the joining of DNA from one chromosome copy to DNA of the other. Crossovers are critical for chromosome separation in the diploid-to-haploid transition, and also promote genetic diversity by shuffling parental genotypes.

In contrast, during mitotic cell growth, crossovers create genome rearrangements and loss of heterozygosity, which are associated with cancer and other diseases. A DNA-unwinding enzyme, called BLM in mammals and Sgs 1 in budding yeast, prevents mitotic crossover recombination by taking apart intermediates that would otherwise give rise to crossovers.

This paper shows that yeast proteins that promote meiotic chromosome synapsis also protect recombination intermediates from Sgs1. If any of these proteins are absent, Sgs 1 prevents both crossover formation and synapsis. These findings show how modulating the activity of a single critical enzyme can either prevent or promote crossover recombination, which threatens genome stability in mitosis but is essential for genome transmission in meiosis.

kinase produce NCO recombinant DNA molecules at normal levels, but lack $\mathrm{COs}$ and accumulate $\mathrm{dHJ}$ intermediates $[16,19]$. Mutants in any of several budding yeast genes (ZIP1, ZIP2, ZIP3, ZIP4/SPO22, MSH4, MSH5, or MER3, referred to here collectively as $Z M M$ genes) also show CO loss without apparent NCO defects (reviewed in [9]). In strains of the SK1 genetic background that are sporulated at $33^{\circ} \mathrm{C}, \mathrm{zmm}$ mutants display severe defects in single-end invasion and $\mathrm{dHJ}$ intermediate formation as well as $\mathrm{CO}$ defects, and this $\mathrm{CO}$ loss is not accompanied by an increase in NCO DNA molecules [17]. This finding is consistent with an earlier suggestion that the $\mathrm{CO} / \mathrm{NCO}$ decision is made at an early step, possibly at or soon after DSB formation [20]. If the decision were made later, blocking $\mathrm{CO}$ formation might allow COdesignated DSBs to be repaired as NCOs, which would result in increased NCO production. These two classes of COdefective mutants also differ in their effect on homolog synapsis, in that $n d t 80$ and $c d c 5$ mutants show normal synapsis [19,21], while $z m m$ mutants display synapsis defects $([17,22]$, and references within).

Of the ZMM proteins, Msh4, Msh5, and Mer3 have known biochemical activities that could stabilize early recombination structures and promote the formation of $\mathrm{dHJ}$ intermediates [23,24]. The other ZMM proteins appear to participate less directly. Zip1 is a major component of the synaptonemal complex (SC) that forms between homolog axes during prophase of meiosis I [7]. It has been suggested that Zip2 and Zip3 are part of a meiosis-specific ubiquitin- or SUMO-conjugating complex [25-27]. In zip1s mutants, homolog axes are no longer tightly paired, but instead associate at a few sites per chromosome that are marked by foci of Zip2 and Zip3 [7,22,28,29]. Accumulating data suggest that, in wild-type budding yeast, these Zip2/Zip3 foci, which also contain Msh4 and Msh5 [30,31], mark sites both of CO recombination and of $\mathrm{Zip} 1$ polymerization initiation
$[22,28,29,32,33]$. These foci, whose protein contents are termed the synapsis initiation complex (SIC), may correspond to the late recombination nodules that mark CO sites in higher eukaryotes [22,34].

Sgs1, a budding yeast RecQ-type helicase, has been implicated in regulating the $\mathrm{CO} / \mathrm{NCO}$ decision and in maintaining genome stability. The absence of Sgs 1 causes increased mitotic recombination [35], especially when mismatches are present in the recombining partners [36,37]. Mutants lacking Sgs1 also show increased chromosomal rearrangement [38] and reduced sporulation efficiency and spore viability [35]. Similarly, vertebrate cells lacking BLM, an Sgs 1 homolog, show elevated rates of sister-chromatid exchange and chromosome rearrangements [39-41]. These mutant phenotypes are consistent with the Sgs1/BLM helicase having a direct anti-CO activity, although many of these observations can also be explained by suggesting that Sgs1/ BLM prevents the formation of lesions that provoke recombination or rearrangement.

Consistent with a role for these helicases in directing events away from COs and towards NCOs, human BLM and TOP3 $\alpha$ together can dissolve synthetic $\mathrm{dHJ}$ substrates in vitro to produce NCOs [42]. While limited solubility has prevented a similar study of Sgs1 [43], two observations support the suggestion that it has anti-CO activity. First, two separate studies, one of spontaneous mitotic recombination and the other of the mitotic repair of a DSB formed by the HO endonuclease, both found about a 2-fold increase in $\mathrm{CO}$ recombinants in sgs 1 mutants relative to wild-type, although the vast majority of repair products in both cases were NCOs $[44,45]$. Second, Rockmill et al. found that, in cells of the BR strain background, the frequency of meiotic COs among tetrads and the number of Zip3-green fluorescent protein (GFP) foci per pachytene nucleus were both about 1.4-fold greater in sgs 1 mutants than in wild-type [32]. Rockmill et al. also showed that sgs 1 mutation accelerates homolog synapsis in otherwise wild-type cells, and restores close, end-to-end association between homolog axes in zipl mutants. They referred to the axial association (AA) seen in zipl sgs 1 as pseudosynapsis, to distinguish it from true synapsis, where end-to-end SC is present.

In order to learn more about the role of Sgs 1 in meiotic recombination, we examined the effect of sgs 1 mutants on meiotic recombination, using both tetrad analysis and an assay that directly scores recombination at the DNA level (Figure 1). Our findings indicate that, in wild-type cells, Sgs 1 activity has a limited role in $\mathrm{CO}$ formation and does not play a unique role in NCO formation. However, in $z \mathrm{~mm}$ mutants, where $\mathrm{CO}$ formation is markedly reduced and synapsis is impaired, sgs 1 mutants restore COs, in some cases to nearly wild-type levels, and also restore tight homolog AA. These data demonstrate that Sgs1 has anti-CO activity, and suggest that an important role for the SICIZMM proteins is to protect nascent $\mathrm{CO}$-designated recombination intermediates from dissolution by Sgs1.

\section{Results}

Previous studies examined sgs $1 \Delta$ and sgs $1 \Delta C 795$ mutants in the BR strain background and found a modest $(0 \%-60 \%)$ increase in allelic crossing over, but did not directly evaluate the effect on NCO recombinants ([32]; B. Rockmill, K. 
A
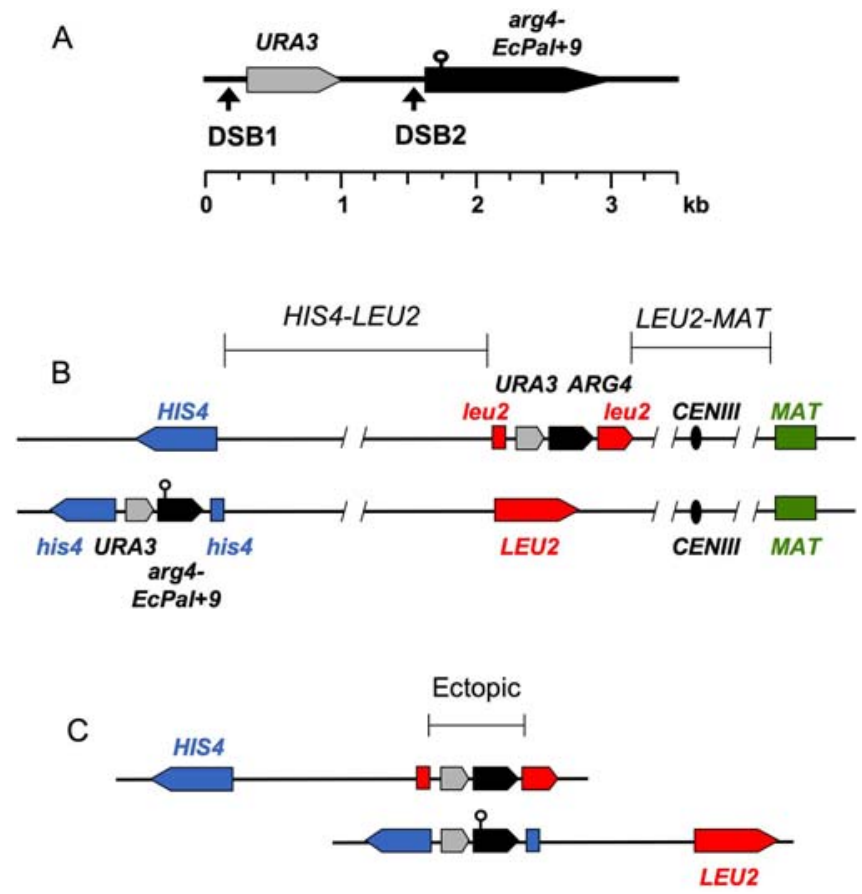

Figure 1. Recombination Interval Used for Molecular Analyses

(A) The 3.5-kilobase ectopic recombination interval contains coding sequences for URA3 (gray) and ARG4 (black). DSBs form in the promoter regions of URA3 (DSB1) and ARG4 (DSB2). The lollipop in arg4 represents a palindromic sequence inserted at +9 of the open reading frame; this mutation is used to score gene conversion [16].

(B) The ectopic recombination interval is inserted at HIS4 (blue) on one copy of Chromosome III and at LEU2 (red) on the homolog. HIS4 and LEU2 are 16.7 kilobases apart. In rad50S strains, where DSBs persist, $5 \%$ of chromosomes have a DSB in his4::URA3-ARG4 and 0.7\% have a DSB in leu2::URA3-ARG4 [16]. The centromere (black circle) and MAT locus (green) are also indicated. Allelic COs can be scored in the HIS4-LEU2 and LEU2-MAT intervals.

(C) Ectopic COs can occur between his4::URA3-ARG4 and leu2::URA3ARG4.

DOI: 10.1371/journal.pgen.0020155.g001

Voelkel-Meiman, and G. S. Roeder, unpublished data). In the SK1 background, homozygous sgs $1 \Delta$ diploids display high chromosome instability, and mating-type heterozygosity cannot be maintained at levels that ensure sporulation in liquid culture. To extend evaluation of the meiotic role of Sgs 1 to SK1 strains, where recombination can be readily scored at the DNA level, we used two sgs 1 mutant alleles, sgs $1 \Delta C 795$ and $s g s 1-m n$. Neither allele displays the same extent of chromosome instability as $\operatorname{sg} 1 \Delta$, and both support efficient premeiotic growth and sporulation. The sgs $1 \Delta C 795$ allele expresses only the first 652 amino acids of the protein and is lacking both the helicase domain [46] and a region called the HRDC domain, which in BLM interacts with Holliday junctions [42]. A DNA fragment containing SGS1 or sgs $1 \Delta C 795$ coding sequences along with 600 nucleotides of upstream sequences was integrated at TRP1 in strains where the endogenous SGS1 gene was deleted (see Materials and Methods). We will refer to strains with sgs $1 \triangle C 795$ at TRP1 as $\operatorname{sgs} 1 \triangle C 795$, and to isogenic control strains with SGS1 at TRP1 as TRP1:SGS1. "Wild-type" will be reserved for strains with $S G S 1$ at its normal locus. In the sgs 1-mn allele, SGS1 is transcribed from a $C L B 2$ promoter, which is expressed during the mitotic cell cycle but not during meiosis. TRP1:SGS1 and
A

Spore Number of viable spores per tetrad

\begin{tabular}{|c|c|c|c|c|c|c|}
\hline & viability $(\%)$ & 4 & 3 & 2 & 1 & 0 \\
\hline Wild type & 98 & 174 & 9 & 3 & 0 & 0 \\
\hline TRP1:SGS1 & 98.7 & 252 & 8 & 3 & 0 & 0 \\
\hline sgs $1-m n$ & 89.8 & 184 & 54 & 15 & 2 & 4 \\
\hline sgs $1 \Delta C 795$ & 82.2 & 107 & 60 & 25 & 6 & 4 \\
\hline TRP1:SGS1 msh4A & 42.8 & 61 & 15 & 73 & 17 & 98 \\
\hline sgs $1 \Delta C 795 \mathrm{msh} 4 \Delta$ & 76.3 & 166 & 93 & 66 & 23 & 12 \\
\hline
\end{tabular}

B

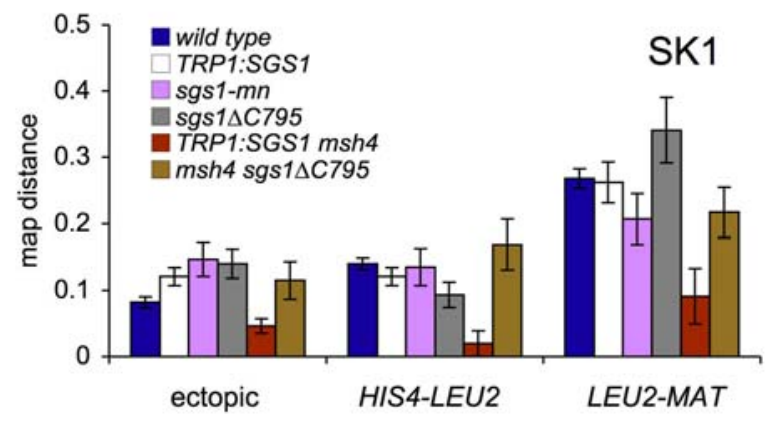

C

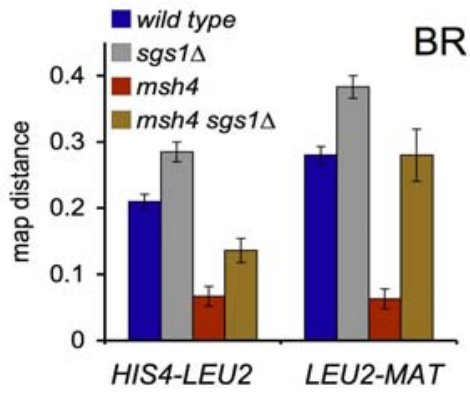

Figure 2. Loss of Full Sgs1 Activity Restores Spore Viability and Crossing Over to $\mathrm{zmm}$ Mutants

(A) Overall spore viability and patterns of spore lethality in tetrads from SK1 strains.

(B) Map distance ( $\mathrm{cM}$; error bars denote standard error of map distance) in three intervals on Chromosome III in SK1 (see Figure 1B and 1C for details). Values for wild-type are from [16].

(C) Map distance in two allelic intervals on Chromosome III in BR strains. Values for wild-type and sgs $1 \Delta$ are from [32].

DOI: 10.1371/journal.pgen.0020155.g002

wild-type strains show similar spore viability ( $\geq 98 \%$; see also Figure 2A) and resistance to methyl methane sulfonate (unpublished data). Both sgs $1 \Delta C 795$ and sgs $1-m n$ show reduced spore viability (Figure 2A), with spore inviability patterns typical of random spore death. A substantial fraction of this spore death is likely to be due to premature separation of sister chromatids, associated with recombination near centromeres (B. Rockmill, K. Voelkel-Meiman, and G. S. Roeder, unpublished data).

Sgs1 Has a Limited Effect on CO Recombination in WildType Cells

We examined meiotic recombination in SK1 strains carrying a 3.5-kilobase URA3-ARG4 recombination interval inserted at his 4 on one copy of Chromosome III and at leu2 on the homolog (Figure 1). COs in three intervals can be scored in these strains: ectopic COs between the his4::URA3-ARG4 and leu2::URA3-ARG4 inserts, allelic COs in the HIS4-LEU2 interval, and allelic COs in the LEU2-MAT interval. 
TRP1:SGS1 strains displayed a 1.4-fold greater frequency of ectopic COs when compared with wild-type $(p<0.001, G$ test), but $\mathrm{CO}$ frequencies in the two allelic intervals were similar to those seen in wild-type (Figure 1B). Both sgs1 mutants produced ectopic COs at frequencies similar to those seen in TRP1:SGS1 and about 1.7-fold greater than in wildtype. Allelic CO frequencies in sgs 1 mutants were not consistently greater or less than those seen in wild-type or TRP1:SGS1, and in all cases were within $30 \%$ of wild-type or TRP1:SGS1 values (Figure 2B). In the BR background, sgs1 $\Delta$ null mutants and sgs $1 \triangle C 795$ display consistent increases of $30 \%-40 \%$ in allelic crossing over in the HIS4-LEU2 and LEU2-MAT intervals [32].

The Zip3 protein forms foci on pachytene chromosomes, and it has been suggested that, in wild-type cells, these foci mark sites of COs [22]. In the BR strain background, sgs1 $\Delta$ null mutants and sgs $1 \Delta C 795$ mutants display a 1.5- and 1.3fold increase, respectively, in the number of Zip3 foci, detected using a Zip3-GFP fusion protein ([32]; B. Rockmill, K. Voelkel-Meiman, and G. S. Roeder, unpublished data). Loss of functional Sgs1 protein has a similar impact on Zip3-focus formation in the SK1 background. Comparable numbers of Zip3-GFP foci were detected in wild-type and TRP1:SGS1 nuclei (wild-type $63 \pm 8$ foci/nucleus, 34 nuclei scored; TRP1:SGS1 $66 \pm 7$ foci/nucleus, 40 nuclei scored), while about $25 \%$ more foci were detected in sgs $1 \Delta C 795$ nuclei $(82 \pm 9$ foci/nucleus, 39 nuclei scored; $p<0.001, t$-test).

Most tetrad analyses consider only data from four-spore viable tetrads. Because COs promote spore viability, this can overestimate $\mathrm{CO}$ frequencies in mutant backgrounds in which spore viability is reduced. To examine every meiotic product regardless of viability, and to determine whether or not Sgs1 function affects NCO formation, we scored recombinants in the ectopic URA3-ARG4 interval at the molecular level, using DNA from SK1 cultures undergoing synchronous meiosis (Figure 3).

Timing of DSB appearance and disappearance, maximal levels of DSBs, and timing of meiotic divisions did not differ substantially among wild-type, TRP:SGS1, sgs1 $\triangle C 795$, and sgs1$m n$ (Figure 3D and unpublished data). Molecular analysis revealed no statistically significant difference among wildtype, TRP1:SGS1, sgs1 $\triangle C 795$, or sgs1-mn strains with regards to the timing of formation or final levels of NCOs or COs in the ectopic recombination interval (Figures 3D and S2), although experiment-to-experiment variation would have obscured CO increases of $30 \%$ or less. These results indicate that, in otherwise wild-type SK1 cells, the majority of CO and NCO recombinant molecules form independently of full Sgs1 function.

We performed a similar analysis in strains containing a different recombination interval, URA3-tel-ARG4, integrated at HIS4 and LEU2 [18]. This interval differs from the URA3$A R G 4$ interval described above in that DSBs occur at a single site in the interval and form more frequently $(20 \%$ versus $5 \%$ of chromosomes). Wild-type, TRP1:SGS1, and sgs1 1 C795 strains produced similar levels of NCO and CO recombinant molecules in this interval (Figure S3).

\section{Sgs1 Inhibits CO Formation in zmm Mutants}

The finding that Sgs1 has a limited impact on meiotic recombination in wild-type SK1 cells stands in contrast to numerous reports suggesting a prominent anti-CO function during the mitotic cell cycle. Because SIC/ZMM proteins promote meiotic $\mathrm{CO}$ formation, we reasoned that they might be masking the anti-CO activity of Sgs1. Therefore, we examined the effect of sgs 1 mutation on meiotic recombination in several $\mathrm{zmm}$ mutants in SK1 and BR strains. The results of these studies are summarized below.

msh4 $\Delta$. SK 1 msh4 mutants show relatively high sporulation frequencies and spore viability [31], allowing tetrad-based measurements of genetic distances. Consistent with previous studies [31], TRP1:SGS1 msh4A strains showed about 2.5-fold fewer COs compared with TRP1:SGS1 MSH4, in all three intervals illustrated in Figure 1, a marked reduction in spore viability, and a disproportionate increase in the number of tetrads with two or no viable spores (Figure 2A and 2B). All three phenotypes were suppressed by sgs $1 \Delta C 795$ (Figure 2), with similar spore viability patterns and $\mathrm{CO}$ frequencies seen in sgs $1 \Delta C 795 \mathrm{MSH} 4$ and $\operatorname{sgs} 1 \Delta C 795 \mathrm{msh} 4 \Delta$. A similar $m s h 4 \Delta$ $\mathrm{CO}$ defect, and suppression by $\operatorname{sgs} 1 \Delta$, was seen in BR strains (Figure 2C).

Molecular assays confirm this $\mathrm{CO}$ defect, and its suppression by sgs 1 mutation. COs in TRP1:SGS1 msh4 4 were reduced 3.7-fold relative to TRP1:SGS1 (Figure 3D). A similar reduction was seen in $m s h 4 \Delta$ (Figure $\mathrm{S} 4$ ). COs were increased nearly to MSH4 levels in sgs $1 \Delta C 795 \mathrm{msh} 4 \Delta$ or sgs1-mn $m s h 4 \Delta$ (2.7-fold greater than TRP1:SGS1 $m s h 4 \Delta$ or $m s h 4 \Delta$ alone; Figures 3 and $\mathrm{S} 4$ ). We found no substantial differences in the time of formation or in final levels of NCO recombinants between $m s h 4 \Delta$ and control strains. The $m s h 4 \Delta$ mutant also had no defects in DSB formation, DSB repair, or meiotic progression.

mer3 $\Delta$. Unlike $m s h 4 \Delta$, mer $3 \Delta$ cells show DSB repair and meiotic progression defects (Figure 3; [47]). DSBs formed normally in both TRP1:SGS1 mer $3 \Delta$ and sgs $1 \Delta C 795$ mer $3 \Delta$ strains, but some breaks persisted beyond the normal time of repair, with DSBs detectable in TRP1:SGS1 mer3 $\Delta$ cells after $12 \mathrm{~h}$ of sporulation. This DSB repair defect was partially suppressed by sgs $1 \Delta C 795$, with all DSBs gone after $10 \mathrm{~h}$ of sporulation. Meiotic progression was also defective in TRP1:SGS1 mer3, with binucleate cells appearing $3 \mathrm{~h}$ later than normal (Figure 3D), and only about $40 \%$ of cells completing meiosis I by $12 \mathrm{~h}$. A greater fraction of sgs $1 \Delta C 795$ mer $3 \Delta$ cells completed at least one division at $12 \mathrm{~h}$, although progression was still delayed.

The CO defect we observed in TRP1:SGS1 mer $3 \Delta$ strains was more severe than the 2- to 3 -fold reduction previously reported [47]. COs could not be detected in TRP1:SGS1 mer $3 \Delta$ mutants $8 \mathrm{~h}$ after initiation of sporulation, a time when COs had reached a maximum in TRP1:SGS1 MER3. Even at $12 \mathrm{~h}$, CO levels were 17 -fold less than in control strains. This CO defect was partially suppressed by sgs $1 \Delta C 795$, and COs in sgs $1 \Delta C 795$ mer $3 \Delta$ were 7 -fold greater than in TRP1:SGS1 mer3 at $12 \mathrm{~h}$. Nevertheless, CO levels reached only about $40 \%$ of the maximum level seen in MER3 controls. A modest NCO defect was also seen in TRP1:SGS1 mer3A. After $8 \mathrm{~h}$ of sporulation, NCO levels in both TRP1:SGS1 mer $3 \Delta$ and $\operatorname{sgs} 1 \Delta C 795$ mer $3 \Delta$ were less than those seen in TRP1:SGS1 $M E R 3$, although NCO frequencies reached or exceeded those seen in TRP1:SGS1 MER3 by $12 \mathrm{~h}$.

zip1 $\Delta$ and zip2 $\Delta$. DSB formation occurred on time, and most DSBs were repaired in $z i p 1 \Delta$ and $z i p 2 \Delta$ strains. These strains also showed delayed meiotic progression that was not affected by sgs $1 \Delta C 795$ (Figure 3 ). At $8 \mathrm{~h}$ after initiation of 


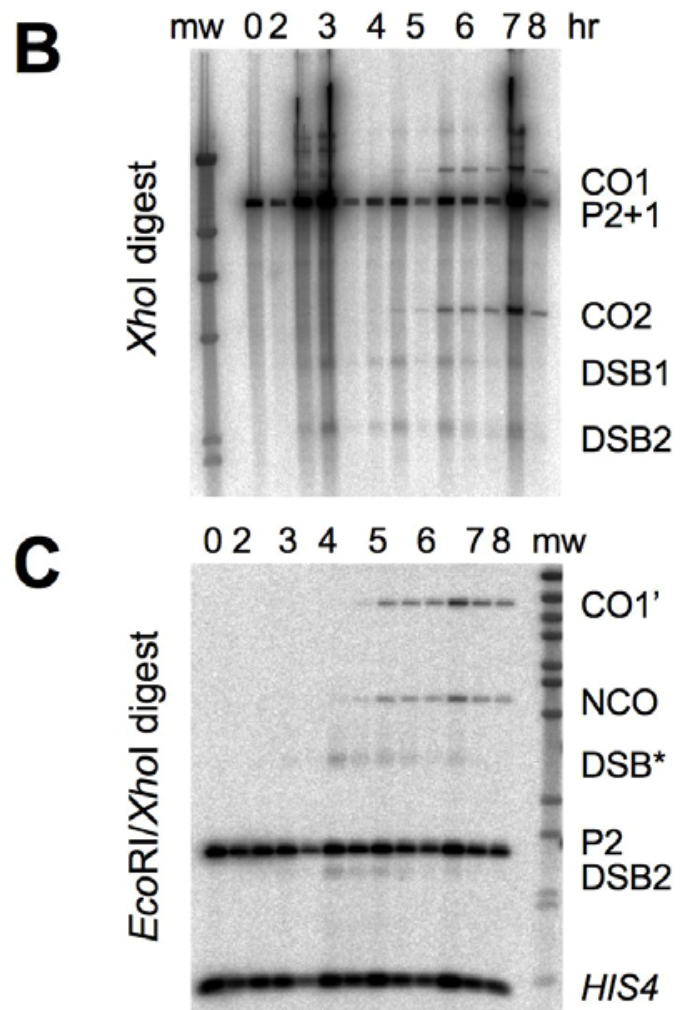

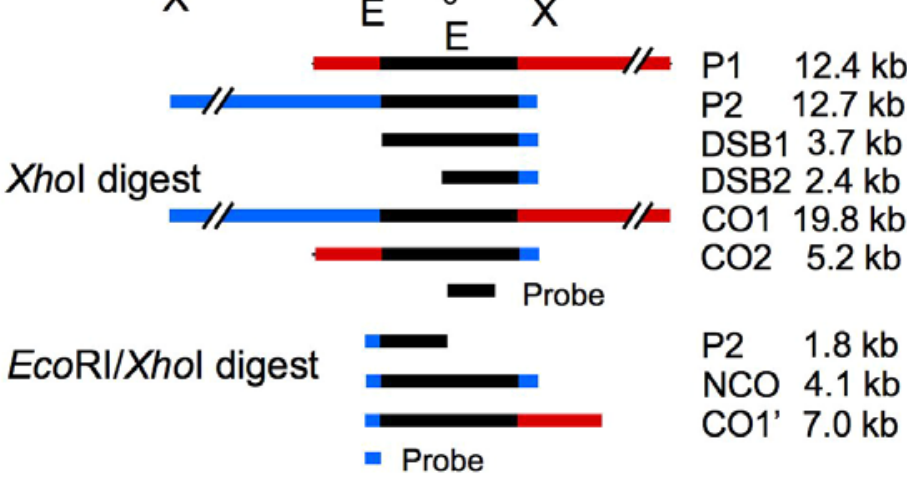

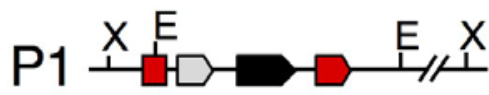

leu2 URA3ARG4leu2

DSB1 DSB2

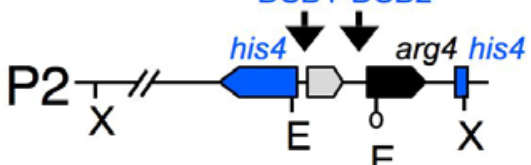

P1 $12.4 \mathrm{~kb}$

P2 $12.7 \mathrm{~kb}$

DSB1 $3.7 \mathrm{~kb}$

DSB2 $2.4 \mathrm{~kb}$

CO1 $19.8 \mathrm{~kb}$

P2 $1.8 \mathrm{~kb}$

NCO $4.1 \mathrm{~kb}$

CO1' $7.0 \mathrm{~kb}$ $\begin{array}{lllllll}02 & 3 & 4 & 5 & 6 & 78 & \mathrm{mw}\end{array}$
D

TRP1:SGS1 msh4 TRP1:SGS1 mgs1 1 C795
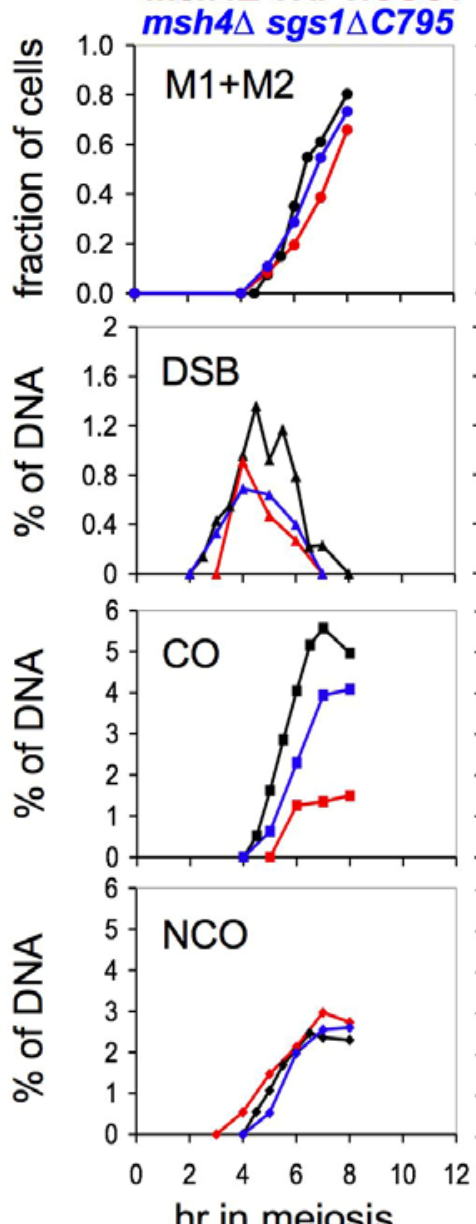

$\mathrm{hr}$ in meiosis
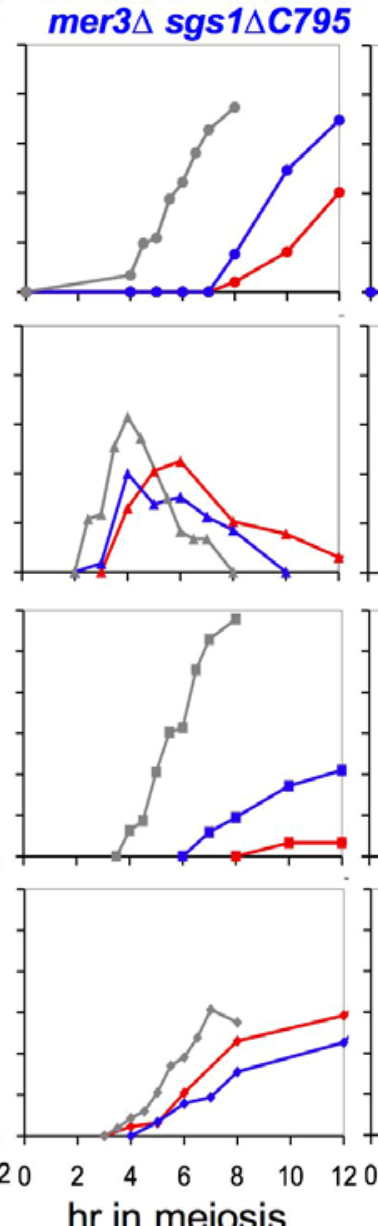

$\mathrm{hr}$ in meiosis
TRP1:SGS1

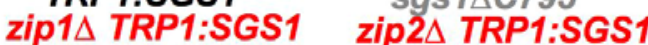
zip1 $\Delta$ sgs1 $\triangle C 795 \quad$ zip2 $\Delta$ sgs1 $\triangle C 795$
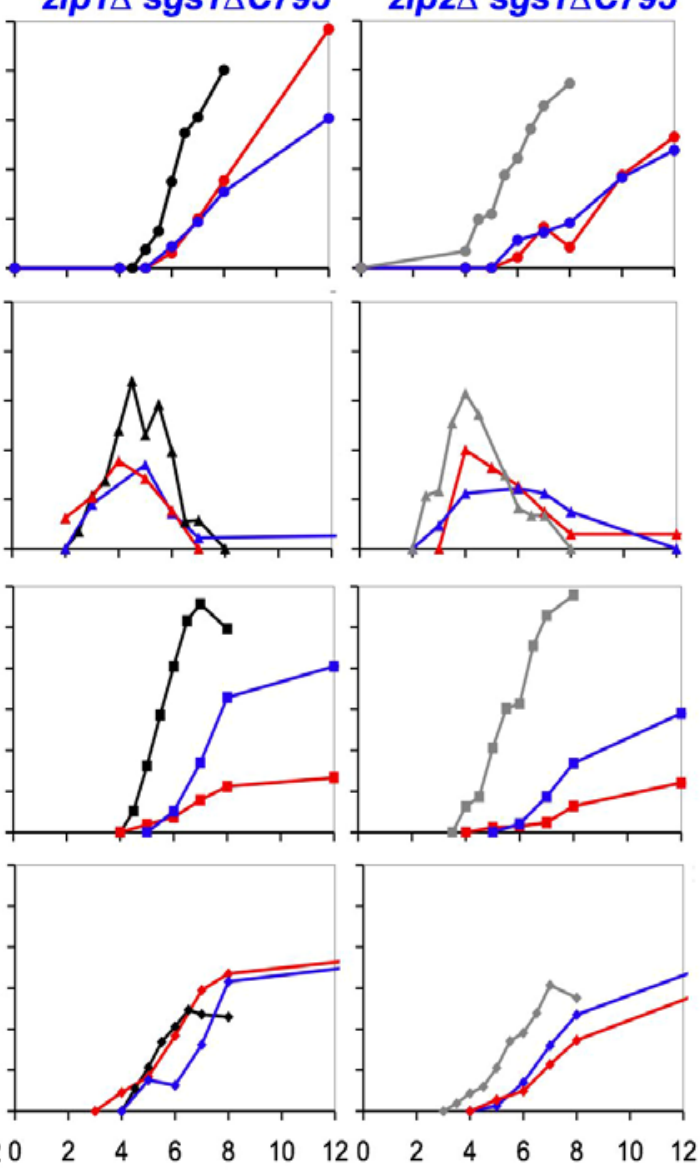

$\mathrm{hr}$ in meiosis 
Figure 3. Sgs1 Prevents COs in zmm Mutants

(A) Schematic representation of the ectopic URA3-ARG4 interval. Symbols are as in Figure 1. EcoRI $(\mathrm{E})$ and Xhol (X) restrictions sites are indicated. To detect COs and DSBs, DNA is digested with Xhol and probed with ARG4 sequences (ArgD; [16]). To detect NCOs and a CO product (CO1'), DNA is digested with EcoRI and Xhol and probed with HIS4 sequences (HisU; [16]).

(B) Southern blot of DNA isolated from a meiotic culture of MJL3035 (TRP1:SGS1) at the indicated time after initiation of sporulation, digested, and probed to detect COs and DSBs. mw, HinDIII digest of phage $\lambda$ DNA. DSBs occur in URA3-ARG4 inserted at both his4 and leu2, but are $\sim 7$-fold stronger in the his4 insert than at leu2 [16]. Palindrome cleavage, by unidentified activities, occurs at the same time as CO formation and results in a band about the size of DSB2 (T. Allers, L. Jessop, and M. Lichten, unpublished data).

(C) Southern blot of the same samples, digested and probed to detect NCO and CO1' recombinants. HIS4, HIS4 locus lacking an insert; DSB*, DSB1 product where resection has passed the EcoRI site in HIS4; mw, BstEll digest of phage $\lambda$ DNA.

(D) Molecular analysis of mutants. Color codes: TRP1:SGS1, black; sgs1 $\triangle C 795$, grey; TRP1:SGS1 zmm single mutants, red; sgs $1 \Delta C 795 \mathrm{zmm}$ double mutants, blue. $M 1+M 2$, percent of cells containing at least two nuclei, a sign of passage through meiosis 1. DSB, DSB1 band signal/total lane signal from Southern blots as in (B). CO, CO1' signal/total lane signal from Southern blots as in (C). NCO, NCO band signal/total insert signal from Southern blots as in (C). DOI: 10.1371/journal.pgen.0020155.g003

sporulation, COs were present in TRP1:SGS1 zip1D at $20 \%$ of the maximum level seen in TRP1:SGS1 ZIP1. The sgs1 $\triangle C 795$ allele partially suppressed this defect, increasing COs 3-fold. NCO formation was unaffected. TRP1:SGS1 zip2D mutants displayed a more severe CO defect. At $8 \mathrm{~h}$, COs were present at $11 \%$ of the maximum seen in TRP1:SGS1 ZIP2. At $12 \mathrm{~h}$, COs levels had increased another 2-fold. COs were increased about 3 -fold by sgs $1 \Delta C 795$ in zip2 $\Delta$, as they were in TRP1:SGS1

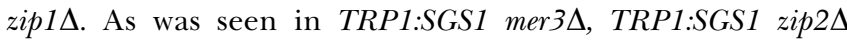
caused a slight delay and reduction in NCOs that was not suppressed by sgs1 $\triangle C 795$; in both zip $2 \Delta$ TRP1:SGS1 and zip2 $\Delta$ sgs $1 \triangle C 795$, NCOs continued to accumulate and at $12 \mathrm{~h}$ their level exceeded the maximum seen in TRP1:SGS1 at the same time (Figure 3).

We also considered whether $\operatorname{sgs} 1 \Delta$ suppresses the allelic CO defect seen in zip1 $\Delta$ in the BR strain background. Because zip1 $\Delta$ mutants sporulate poorly in $\mathrm{BR}$, map distances were measured by random spore analysis; $\operatorname{sgs} 1 \Delta$ caused about a 2 fold increase in COs in both intervals (Table S2).

In summary, in the conditions used in these experiments, $z m m$ mutants differ in terms of meiotic progression, DSB repair, and $\mathrm{CO}$ formation defects, consistent with the diversity of defects previously seen when SK1 $\mathrm{zmm}$ mutants are sporulated at $23{ }^{\circ} \mathrm{C}$ [17]. Despite these differences, in all cases the $z \mathrm{~mm}$ mutant phenotype is at least partially suppressed by loss of Sgs1.

\section{Sgs1 Inhibits Homolog AA in mer $3 \Delta$ and zip $3 \Delta$ Mutants}

Despite the absence of Zip1, the protein that normally occupies the space between synapsed homolog axes [48], close juxtaposition of homolog axes along their lengths is observed in sgs 1 zip1 double mutants in both BR and SK1 strain backgrounds ([32] and unpublished data). To determine whether this pseudosynapsis occurs in other zmm sgs 1 double mutants, we examined chromosome morphology in surfacespread meiotic nuclei, using antibodies against Zip1 and antibodies against Red1, a major component of meiotic chromosome axes. In wild-type yeast, chromosome cores never achieve fully continuous Red1 staining [49]. However, in synapsis-defective $z m m$ mutants, Red1 accumulates and localizes continuously along each chromosome axis [29]. Thus, Red1 staining provides a means to visualize chromosome contours in the absence of Zip1 staining.

In SK1 strains, both TRP1:SGS1 and sgs1 $1 C 795$ displayed normal chromosome morphology, with axes of homologous chromosomes closely juxtaposed and continuous end-to-end Zip1 staining (unpublished data). However, both strains displayed an increased frequency of polycomplexes (extrachromosomal arrays of SC components) compared with wild- type (28/83 nuclei in TRP1:SGS1 and 34/74 nuclei in sgs $1 \Delta C 795$ versus $4 / 50$ nuclei in wild-type after $5 \mathrm{~h}$ of sporulation). This increase in polycomplex formation suggests a modest defect or delay in SC formation.

TRP1:SGS1 mer3A diploids displayed a severe synapsis defect, with about $90 \%$ of nuclei showing separated homolog axes (as evidenced by Red1 staining) and little Zip1 localization (Figure 4). Homolog AA was partially restored in mer3 3 sgs $1 \Delta C 795$ mutants, with more than $90 \%$ of cells displaying either some or all chromosomes with axes in close alignment along their lengths (Figure 4A). These fully aligned chromosomes displayed only low levels of discontinuous Zip1 staining, in contrast to the end-to-end continuous staining seen in sgs 1 single mutants and in wild-type $([7,17,32]$ and unpublished data). Although about half of mer $3 \Delta \operatorname{sgs} 1 \Delta C 795$ nuclei displayed full pseudosynapsis of homologs, an equal number displayed partial pseudosynapsis, where only some homologs or parts of homologs appeared pseudosynapsed.

We also determined the effect of Sgs 1 on synapsis in $z i p 3 \Delta$ BR strains, which display a relatively mild CO and progression defect [28]. Only a small fraction of zip3 mutant cells displayed full homolog AA, and this occurred at relatively late times in meiosis. Despite this synapsis defect, about $75 \%$ of zip $3 \Delta$ mutant cells progress to form mature asci (unpublished data). Deletion of $S G S 1$ from zip $3 \Delta$ mutants restored full homolog AA to more than half of nuclei (Figure 4B). Of the chromosome pairs in which axes were closely juxtaposed, most were truly synapsed (i.e., displayed end-to-end Zip1 staining) and a minor fraction were pseudosynapsed (Figure 4B and unpublished data).

\section{Discussion}

In previous studies of BR strains, Rockmill et al. showed that the $\operatorname{sgs} 1 \Delta$ and $\operatorname{sgs} 1 \Delta C 795$ mutations increase crossing over in allelic intervals (1.2- to 1.4-fold) and cause a corresponding increase (1.3- to 1.4-fold) in the number of Zip3 foci, which are thought to be cytological markers of CO sites [32]. In this study, we examined the effect of sgs 1 mutations on meiotic recombination in SK1 strains, using assays that detected NCO and CO DNA molecules produced by ectopic recombination. We did not observe a statistically significant increase in CO molecules in two test intervals, although experiment-to-experiment variation would have obscured an increase of $30 \%$ or less. We also did not observe a consistent increase in $\mathrm{CO}$ recombination, measured by tetrad analysis in three genetic intervals on Chromosome III. We did observe an increase (by about 25\%) in Zip3 foci in sgs1 $\triangle C 795 \mathrm{SK} 1$ strains (relative to TRP1:SGS1). If Zip3 foci are 
A

minimal
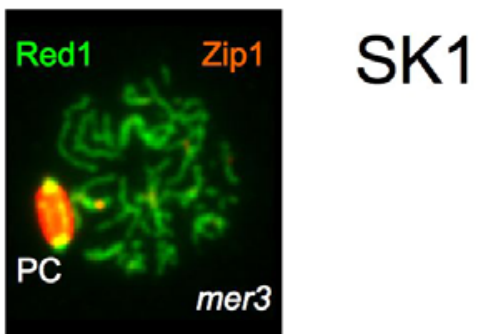

partial
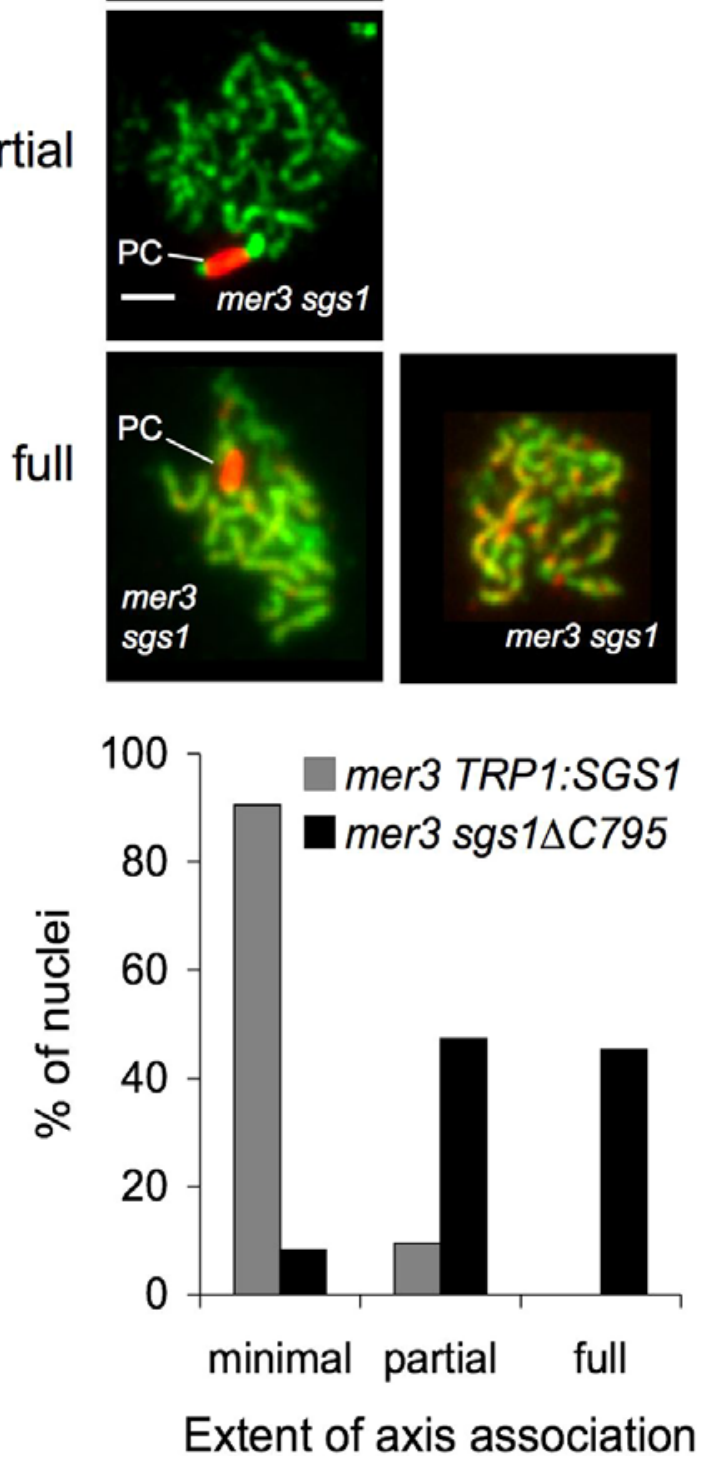

B

minimal
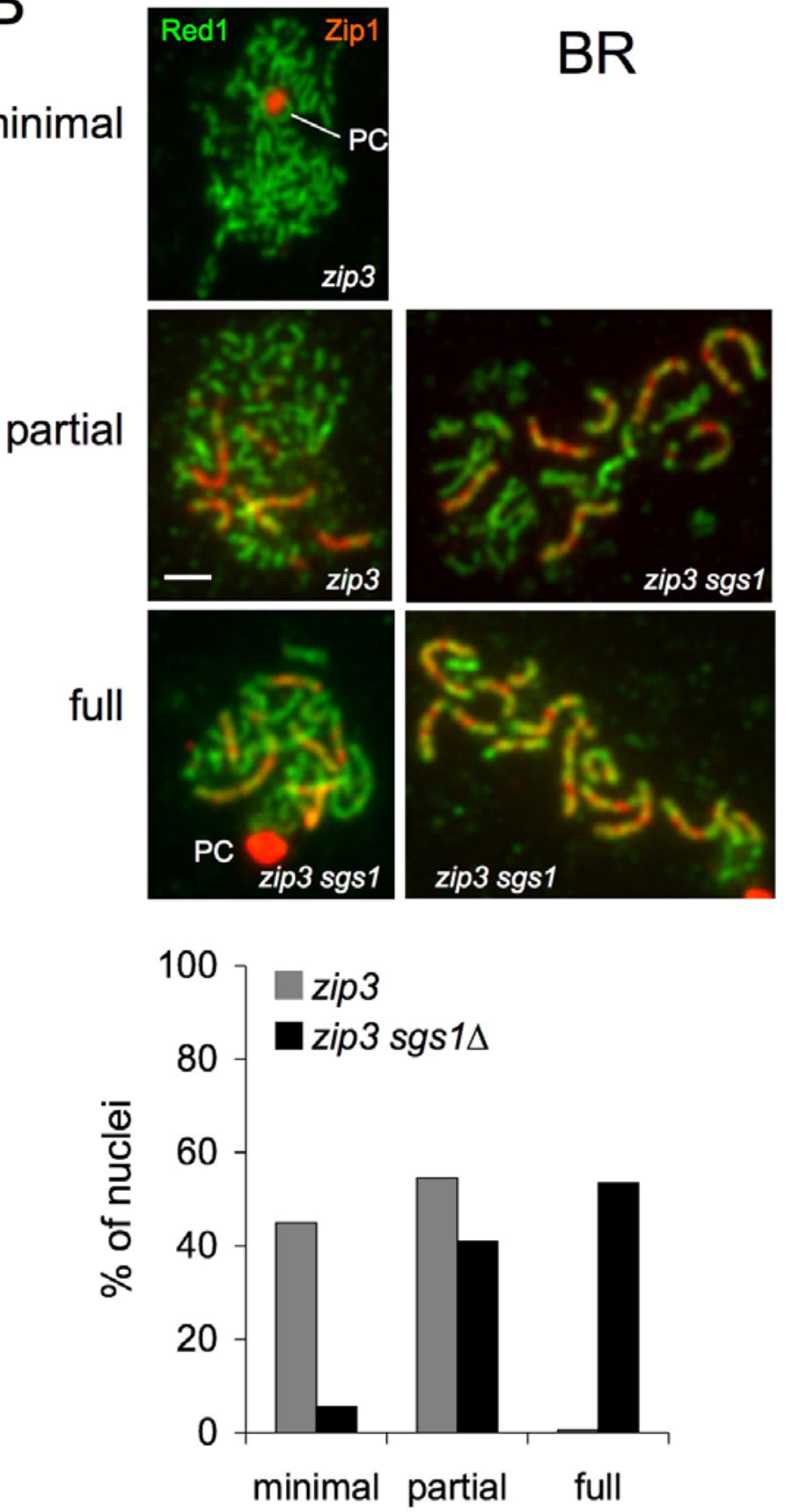

Extent of axis association

Figure 4. Sgs1 Prevents $A A$ in mer3 $\Delta$ and zip3 $\Delta$ Mutants

(A) Analysis of AAs in TRP1:SGS1 mer3 $\Delta$ and mer3 sgs1 $\Delta$ C795 SK1 strains. Nuclei from cells harvested $5 \mathrm{~h}$ after initiation of sporulation were surface spread and probed with anti-Zip (red) and anti-Red1 (green) antisera. Nuclei where chromosomes displayed linear Red1 were examined and classified as displaying minimal, partial, or full pseudosynapsis, as described in Materials and Methods. Nuclei with fully associated chromosomes displayed discontinuous Zip1 staining (right-hand example), Zip1 localization in polycomplexes (PC, left-hand example), or both. White bar: 2 microns.

(B) Analysis of AAs in zip3 $\Delta$ and zip3 $\Delta$ sgs $1 \Delta$ BR strains. Nuclei from cells harvested $18 \mathrm{~h}$ after the initiation of sporulation were spread, stained, and analyzed as for mer3 strains. In most nuclei with full AA, at least some chromosome pairs displayed end-to-end Zip1 staining with the remainder being pseudosynapsed.

DOI: 10.1371/journal.pgen.0020155.g004

accurate markers of CO sites, this finding would be consistent with a modest increase in crossing over on a genome-wide basis. In a separate study, Oh and Hunter have examined the effect of $s g s 1 \Delta C 795$ on CO recombination in SK1 diploids, using both genetic and molecular assays. Their data indicate that, in the SK1 background, loss of SGS1 function increases overall CO frequencies by no more than $20 \%$ (S. Oh and N. Hunter, personal communication). 
We also found that, in SK1 sgs1 mutants, NCO recombinants are recovered at frequencies similar to those seen in wild-type; similar results have been observed in BR strains [32]. Thus, the bulk of the genetic, DNA-based, and cytological assays suggest that, in wild-type cells, there must be proteins other than Sgs1 that prevent COs, and that promote NCO recombination.

\section{Sgs1 Mediates the CO Defect in zmm Mutants}

COs are required for proper chromosome segregation during meiosis, and both meiotic CO distributions and the time of their formation are tightly regulated in most organisms [9,50]. It is therefore likely that the anti-CO activity of the Sgs1/BLM helicase evident in mitotic cells [35-37,39-41] must be modulated during meiosis to allow high CO levels, either by reducing helicase activity or by preventing access to substrates. Our current study identifies SIC/ZMM proteins encoded by the budding yeast ZIP1, ZIP2, ZIP3, MER3, and MSH4 genes, as modulators of the impact of Sgs1. Although mutations in these genes reduce $\mathrm{CO}$ formation, the severity of this defect can vary widely. For example, under the sporulation conditions used in our study, this CO defect ranged from about a 4-fold reduction (in $m s h 4 \Delta$ ) to a virtual elimination (in $m e r 3 \Delta$ ). These differences most likely reflect the different biochemical and structural roles played by the different ZMM proteins, either individually or as part of a larger complex. Nevertheless, the CO defect in these mutants was at least partially suppressed by the loss of Sgs 1 activity. In molecular assays, CO restoration was not accompanied by a corresponding decrease in NCO recombinants, and COs were not restored to levels seen in wild-type or in the sgs $1 \Delta C 795$ single mutant. Genetic assays, in both SK1 and BR backgrounds, also showed substantial restoration of COs to $z \mathrm{~mm}$ mutants by sgs 1 mutation. The sgs $1 \Delta C 795$ mutation also has been found, in genetic and molecular assays, to restore crossing over to $m s h 5$ and $m l h 3$ mutants in the SK1 background (S. Oh and N. Hunter, personal communication). These findings indicate that Sgs1 can act specifically to prevent CO formation during meiosis, but that this activity is primarily manifest in cells lacking intact SIC/ZMM protein function. Below, we briefly consider mechanisms by which Sgs1 might decrease COs in $z m m$ mutants.

COs restored in $z m m$ sgs 1 double mutants come from NCOs. Allers and Lichten suggested that CO and NCO recombinants are the products of alternate processing of an early strand invasion intermediate [16]. Helicase-driven destabilization of this intermediate would produce NCOs via a process similar to synthesis-dependent strand annealing; stabilization would allow capture of the second break end, producing a $\mathrm{dHJ}$ intermediate that subsequently would be resolved as a CO. This model predicts that $\mathrm{CO}$ increases in zmm sgs 1 mutants should be accompanied by equivalent decreases in NCOs. This prediction is not supported. There is no decrease in NCO levels in msh4 sgs 1 and zip1 sgs 1 strains compared with msh4 and zipl single mutants, respectively. The slight decrease in NCOs in mer 3 sgs 1 and zip2 sgs 1 strains compared with the mer 3 and zip2 single mutants, respectively, cannot account completely for the restoration of COs (Figure 3). We therefore consider the alternate processing hypothesis to be unlikely.
COs in sgs1 zmm double mutants depend on Mus81/Mms4. It has been suggested that, in $S$. cerevisiae, most meiotic COs are ZMM-dependent, with a minor fraction being produced by a ZMM-independent pathway that requires Mus81/Mms4 endonuclease activity to resolve recombination intermediates as COs $[51,52]$. One way to account for partial CO restoration in sgs $1 \mathrm{zmm}$ double mutants would be to suggest that Sgs1 activity blocks this ZMM-independent pathway. If this putative second pathway were completely separate from ZMM-dependent processes, then all sgs $1 \mathrm{zmm}$ mutants should display a similar increase in COs, which we do not observe. However, our data do not exclude the possibility that, in the absence of Sgs 1 activity, resolution of intermediates as COs requires Mus81/Mms4 activity. Experiments to test this possibility are ongoing.

ZMM proteins protect pre-CO intermediates from Sgs1. ZMM proteins colocalize in foci whose number and distribution are similar to those of meiotic COs, and it has been suggested that these foci correspond to the late recombination nodules observed in higher eukaryotes that mark sites of crossing over [22]. One possible function for these large structures would be to promote progression of recombination intermediates that are designated to produce COs [17], perhaps by shielding them from Sgs 1 and other helicases, or by modifying Sgs1 so that it no longer has anti-CO activity. If this were the case, then the presence or absence of either ZMM proteins or fully functional Sgs1 should not substantially alter NCO levels. Our data are consistent with this suggestion, and thus provide further support for previous suggestions that NCOs and COs diverge at a very early step in meiotic recombination, and that only limited crosstalk occurs between the two pathways once the separation occurs $[17,20]$.

What happens to the DSBs and recombination intermediates that would have given rise to COs in $z m m$ mutants? Some of these mutants display persistent unrepaired DSBs, which could come either from intermediates disassembled in vivo, or from intermediates, arrested at the strand invasion stage, that fall apart during DNA preparation. However, this is not universally true. In $m s h 4 \Delta$ at $30^{\circ} \mathrm{C}, \mathrm{COs}$ are reduced significantly, yet DSBs do not visibly persist, and cells progress normally through both meiotic divisions. In this case, the DSBs that would have given rise to COs must have been repaired. Because COs are reduced, and NCOs are not increased in this mutant, it is likely that these "missing breaks" are repaired by sister-chromatid recombination, which would be undetected in our assays.

\section{Sgs1 Inhibits Interhomolog AA}

In zip1 single mutants, the cores of each pair of homologous chromosomes are connected at only a few sites, referred to as AAs [7,32]. The number of AAs is increased in zip1 sgs 1 double mutants to the point where homolog axes appear to be tightly associated along their lengths [32]. This sgs1 effect appears to be a general phenomenon for $\mathrm{zmm}$ mutants, as increased association between homolog axes occurs in mer $3 \Delta$ sgs $1 \Delta C 795$ and zip3 sgs $1 \Delta$ double mutants (this study), and in zip2 sgs 1 and zip4 sgs 1 double mutants (B. Rockmill and G. S. Roeder, unpublished data). Zip1 is present in these mutants, and the additional homolog association promoted by $\operatorname{sg} 1$ mutation is often accompanied by regions of normal synapsis (i.e., Zip1 assembly), although synapsis is frequently incomplete. 
A previous study has reported that, in zip1 sgs 1 double mutants, the increase in the number of AAs appears to be much greater than the increase in the number of COs [32]. Our cytological study of mer3 3 TRP1:SGS1 and mer $3 \Delta$ sgs $1 \Delta C 795$ provides further evidence for a discrepancy between the number of COs and the number of AAs. In particular, mer $3 \Delta$ sgs $1 \Delta C 795$ double mutants show complete pseudosynapsis in about half of cells, but COs are restored to levels that are only 1.4-fold greater than those seen in zip1 $\Delta$ TRP1:SGS1 (Figure 3 and unpublished data), where axes associate at only a few points per chromosome. It therefore appears likely that, when full Sgs1 activity is absent, AAs occur at more sites than the ones that give rise to COs.

The molecular nature of the interhomolog interactions at these association sites remains to be determined. However, it is likely that it involves recombination intermediates, since the formation of AAs depends on DSBs [53] and AAs are observed in SK1 strains at a time when most mature CO products have not yet appeared (Figure 3). Because the number of AAs seen in zip1 single mutants approximates the number of COs seen in wild-type [29], it is unlikely that the additional AAs seen in zip $1 \Delta$ sgs $1 \Delta C 795$ or in mer $3 \Delta$ sgs $1 \Delta C 795$ double mutants reflect interhomolog interactions that will eventually be processed to form COs. Instead, we suggest that they contain recombination intermediates that either are resolved as NCOs, or are disassembled and repaired by sisterchromatid recombination later in meiosis. Further study will be required to determine which of these suggestions is correct.

In summary, the data presented here point to a mutual antagonism between Sgs1 and functions that promote homologous chromosome colocalization and synapsis during meiosis. We suggest that, on one hand, the SIC/ZMM proteins prevent Sgs1 from disassembling nascent CO-designated intermediates; on the other hand, Sgs1 activity may limit stable, long-lived associations between homologous chromosomes to sites that will be used for COs.

\section{Materials and Methods}

Strains and media. Strains used for molecular analyses (Table S1A) are all direct derivatives of SK1 [54]. The URA3-ARG4 recombination interval used has been described previously [16]. Strain construction details are given in Protocol S1. Deletions of SGS1, MSH4, MER3, ZIP1, and ZIP2 were made by replacing coding sequences with a G418-resistance cassette [55]. Strains with sgs $1 \Delta C 795$ contain this allele integrated at TRP1 and the endogenous SGS1 locus deleted; as controls, strains with SGS1 at TRP1 were used. The meiotic null allele of SGS1 (sgs 1-mn) was made as described [56]. These sgs1-mn mutants grow as well as wild-type in the presence of $0.012 \%$ MMS, which prevents growth of $s g s 1$-null mutants, indicating that $s g s 1-m n$ retains normal mitotic function. Quantitative Western blots showed that the 3HA-Sgs 1 protein expressed from sgs 1-mn is rapidly degraded during meiosis: $2 \mathrm{~h}$ after induction of sporulation, about $10 \%$ of the protein remains, and none remains after $4 \mathrm{~h}$ (Figure S1).

Zip3 foci were quantified in strains that were heterozygous for an insertion of pSA219 (ZIP3-GFP-URA3; [28]). Strains homozygous for this insert displayed greater than normal spore lethality, and were therefore not used.

Genetic and cytological analyses were also done with strains isogenic to BR1919-8B (Table S1B). Wild-type, $\operatorname{sgs} 1:: K A N$, zip3::URA3, and $m s h 4:: A D E 2$ strains have been described [22,32]. Genetic crosses were used to make double mutants.

Genetic and molecular analyses. Yeast media and genetic procedures were as described [18]. Genetic distance determinations (map distance in $\mathrm{cM}$ and standard error of map distance) used the calculator at http://www.molbio.uoregon.edu/ fstahl. Only tetrads with four viable spores were considered. $G$-test analysis of tetrad class distributions used to calculate map distances used a Microsoft Excel calculator kindly supplied by E. Hoffmann and R. Borts. Recombination intermediates and recombination products were detected and quantified as described [16,18].

Cytological analysis. Nuclear spreads were performed as described [29], with antibody staining and Zip3 focus quantification as described [22,28,32]. In mer $3 \Delta$ and mer $3 \Delta \operatorname{sgs} 1 \Delta C 795$ strains, Zip1 does not assemble properly into SC ([17] and this paper), so chromosome association was evaluated by examining nuclear spreads in which Red1 staining was continuous. AAs were scored as "minimal" if chromosomes consisted of thin (i.e., single) axes with only a few points of association, as "full" if the majority of chromosome axes were thick (i.e., clearly doubled), and as "partial" if they displayed a morphology intermediate between these two states (see Figure 4). 200 nuclei were scored for each mutant genotype.

\section{Supporting Information}

Figure S1. sgs1-mn Is a Meiosis-Specific Null Allele

(A) Western blot probed with anti-HA (top panel) to detect 3HA-Sgs1 expressed from the CLB2 promoter, or anti-Tub2 (bottom panel) to detect Tub2 as a loading control. Protein was extracted from a synchronously sporulating culture of MJL3091 at the indicated times. * indicates cross-reacting protein that is present in all samples.

(B) Graph of relative 3HA-Sgs 1 levels, with $0 \mathrm{~h}$ sample levels set at $100 \%$. This corresponds to between 1 and 1.5 times the level of Sgs 1 seen in $0 \mathrm{~h}$ samples from wild-type cells (unpublished data).

Found at DOI: 10.1371/journal.pgen.0020155.sg001 (1.0 MB TIF).

Figure S2. Effect of $\operatorname{sgs} 1 \Delta C 795$ on CO Recombination in SK1 Strains $\mathrm{CO}$ recombination was measured as described in Figure 3B. Values reflect averages of 7 and $8 \mathrm{~h}$ samples from multiple blots of DNA from several independent cultures. Number of determinations were as follows: wild-type, 18 measurements, 5 cultures; TRP1::SGS1, 8 measurements, 3 cultures; sgs $1 \Delta C 795,6$ measurements, 3 cultures; sgs 1-mn, 5 measurements, two cultures.

Found at DOI: 10.1371/journal.pgen.0020155.sg002 (330 KB TIF).

Figure S3. $\operatorname{sgs} 1 \Delta C 795$ Does Not Substantially Alter CO or NCO Recombination in a Second Interval

$\mathrm{CO}$ and NCO recombination were measured in a URA3-tel-ARG4 recombination reporter insert [8] at LEU2 and HIS4 on parental homologs.

(A) Structure of the insert and detection of recombinants. In this insert, URA3 and ARG4 are in opposite orientations, and recombination is initiated at a single DSB site, promoted by a 60 nucleotide insert containing telomere repeats $(\mathrm{tel})$. $\mathrm{CO} 1$ and $\mathrm{NCO}$ recombinants were detected essentially as described in Figure 3, by digesting and probing as follows: CO1: XhoI digest, probe with ARG4 sequences (black box); NCO: EcoRI/XhoI digest, probe with his4' sequences (blue box).

(B) Average $\mathrm{CO}$ and NCO product frequencies from 7 and $8 \mathrm{~h}$ samples for wild-type (MJL2984), TRP1::SGS1 (MJL3033), and sgs $\triangle C 795$ (MJL3034) strains. Bars indicate standard deviations for the following number of determinations: wild-type: CO 4, NCO 2; TRP1::SGS1: CO 3, NCO 4; sgs $\Delta C 795$ : CO 4, NCO 3.

Found at DOI: 10.1371/journal.pgen.0020155.sg003 (924 KB TIF).

Figure S4. An sgs1 Meiotic Null Mutant Restores COs to $m s h 4 \Delta$ Mutants

Cultures of $m s h 4 \Delta$ (MJL3120, red), sgs1-mn (MJL3091, black), and $m s h 4 \Delta$ sgs1-mn (MJL3124, blue) were sporulated, and samples taken at the indicated times were analyzed for nuclear divisions (MI + MII), DSBs, and $\mathrm{CO}$ and $\mathrm{NCO}$ recombinants ( $\mathrm{NCO}$ and $\mathrm{CO}^{\prime}$ ) as in Figure 3C.

Found at DOI: 10.1371/journal.pgen.0020155.sg004 (719 KB TIF).

Protocol S1. Supplementary Online Methods

Found at DOI: 10.1371/journal.pgen.0020155.sd001 (37 KB DOC).

Table S1. Strain Genotypes

Found at DOI: 10.1371/journal.pgen.0020155.st001 (77 KB DOC).

Table S2. $\operatorname{sgs} 1 \Delta$ Restores Crossovers to a zip1 $1 \Delta$ Mutant in the BR Strain Background

Found at DOI: 10.1371/journal.pgen.0020155.st002 (32 KB DOC). 


\section{Accession Numbers}

The UniProt (http://www.pir.uniprot.org) accession numbers for the proteins mentioned in this paper are BLM helicase (P54132), Cdc5 (P32562), Mer3/Hfm1 (P51979), Msh4 (P40965), Msh5 (Q12175), Ndt80 (P38830), Red1 (P14291), Sgs1 (P35187), Spo11 (P23179), TOP3 alpha (Q13472), Tub2 (P02557), Zip1 (P31111), Zip2 (P53061), Zip3/Cst9 (Q06032), and Zip4/Spo22 (P40511).

\section{Acknowledgments}

We thank E. Hoffmann, S. Brill, R. H. Borts, M. Basrai, and A. Amon for strains, reagents, and statistical tools, C. Mann for technical advice, and D. Chattoraj and Y. Rong for comments that improved

\section{References}

1. Strathern JN, Shafer BK, McGill CB (1995) DNA synthesis errors associated with double-strand-break repair. Genetics 140: 965-972.

2. Paques F, Haber JE (1999) Multiple pathways of recombination induced by double-strand breaks in Saccharomyces cerevisiae. Microbiol Mol Biol Rev 63: 349-404.

3. Jones GH (1987) Chiasmata. In: Moens PB, editor. Meiosis. Orlando (Florida): Academic Press. pp. 213-244.

4. Nicklas RB (1997) How cells get the right chromosomes. Science 275: 632 637.

5. Petronczki M, Siomos MF, Nasmyth K (2003) Un ménage à quatre: The molecular biology of chromosome segregation in meiosis. Cell 112: 423440 .

6. Klapholz S, Waddell CS, Esposito RE (1985) The role of the SPO11 gene in meiotic recombination in yeast. Genetics 110: 187-216.

7. Sym M, Engebrecht JA, Roeder GS (1993) ZIP1 is a synaptonemal complex protein required for meiotic chromosome synapsis. Cell 72: 365-378.

8. Koehler KE, Hawley RS, Sherman S, Hassold T (1996) Recombination and nondisjunction in humans and flies. Hum Mol Genet 5: 1495-1504.

9. Bishop DK, Zickler D (2004) Early decision; meiotic crossover interference prior to stable strand exchange and synapsis. Cell 117: 9-15.

10. Alani E, Padmore R, Kleckner N (1990) Analysis of wild-type and rad50 mutants of yeast suggests an intimate relationship between meiotic chromosome synapsis and recombination. Cell 61: 419-436.

11. Baudat F, Manova K, Yuen JP, Jasin M, Keeney S (2000) Chromosome synapsis defects and sexually dimorphic meiotic progression in mice lacking Spo11. Mol Cell 6: 989-998.

12. Keeney S, Giroux CN, Kleckner N (1997) Meiosis-specific DNA doublestrand breaks are catalyzed by Spo11, a member of a widely conserved protein family. Cell 88: 375-384.

13. Sun H, Treco D, Szostak JW (1991) Extensive 3'-overhanging, singlestranded DNA associated with the meiosis-specific double-strand breaks at the ARG4 recombination initiation site. Cell 64: 1155-1161.

14. Hunter N, Kleckner N (2001) The single-end invasion: An asymmetric intermediate at the double-strand break to double-Holliday junction transition of meiotic recombination. Cell 106: 59-70.

15. Schwacha A, Kleckner N (1995) Identification of double Holliday junctions as intermediates in meiotic recombination. Cell 83: 783-791.

16. Allers T, Lichten M (2001) Differential timing and control of noncrossover and crossover recombination during meiosis. Cell 106: 47-57.

17. Börner GV, Kleckner N, Hunter N (2004) Crossover/noncrossover differentiation, synaptonemal complex formation, and regulatory surveillance at the leptotene/zygotene transition of meiosis. Cell 117: 29-45.

18. Jessop L, Allers T, Lichten M (2005) Infrequent co-conversion of markers flanking a meiotic recombination initiation site in Saccharomyces cerevisiae. Genetics 169: 1353-1367.

19. Clyne RK, Katis VL, Jessop L, Benjamin KR, Herskowitz I, et al. (2003) Pololike kinase Cdc5 promotes chiasmata formation and cosegregation of sister centromeres at meiosis I. Nat Cell Biol 5: 480-485.

20. Storlazzi A, Xu L, Schwacha A, Kleckner N (1996) Synaptonemal complex (SC) component Zip1 plays a role in meiotic recombination independent of SC polymerization along the chromosomes. Proc Natl Acad Sci U S A 93: 9043-9048.

21. Xu L, Ajimura M, Padmore R, Klein C, Kleckner N (1995) NDT80, a meiosisspecific gene required for exit from pachytene in Saccharomyces cerevisiae. Mol Cell Biol 15: 6572-6581.

22. Fung JC, Rockmill B, Odell M, Roeder GS (2004) Imposition of crossover interference through the nonrandom distribution of synapsis initiation complexes. Cell 116: 795-802.

23. Mazina OM, Mazin AV, Nakagawa T, Kolodner RD, Kowalczykowski SC (2004) Saccharomyces cerevisiae Mer3 helicase stimulates $3^{\prime}-5^{\prime}$ heteroduplex extension by Rad51; implications for crossover control in meiotic recombination. Cell 117: 47-56.

24. Snowden T, Acharya S, Butz C, Berardini M, Fishel R (2004) hMSH4-hMSH5 recognizes Holliday junctions and forms a meiosis-specific sliding clamp that embraces homologous chromosomes. Mol Cell 15: 437-451.

25. Perry J, Kleckner N, Borner GV (2005) Bioinformatic analyses implicate the the manuscript. We also thank S. Oh and N. Hunter for communicating results prior to publication.

Author contributions. LJ, BR, GSR, and ML conceived and designed the experiments. LJ, BR, and ML performed the experiments. LJ, BR, GSR, and ML analyzed the data, contributed reagents/ materials/analysis tools, and wrote the paper.

Funding. This work was supported in part by the Intramural Research Program of the NIH, National Cancer Institute, Center for Cancer Research (ML laboratory) and by the Howard Hughes Medical Institute (GSR laboratory).

Competing interests. The authors have declared that no competing interests exist.

collaborating meiotic crossover/chiasma proteins Zip2, Zip3, and Spo22/ Zip4 in ubiquitin labeling. Proc Natl Acad Sci U S A 102: 17594-17599.

26. Hooker GW, Roeder GS (2006) A Role for SUMO in meiotic chromosome synapsis. Curr Biol 16: 1238-1243.

27. Cheng CH, Lo YH, Liang SS, Ti SC, Lin FM, et al. (2006) SUMO modifications control assembly of synaptonemal complex and polycomplex in meiosis of Saccharomyces cerevisiae. Genes Dev 20: 2067-2081.

28. Agarwal S, Roeder GS (2000) Zip3 provides a link between recombination enzymes and synaptonemal complex proteins. Cell 102: 245-255.

29. Chua PR, Roeder GS (1998) Zip2, a meiosis-specific protein required for the initiation of chromosome synapsis. Cell 93: 349-359.

30. Novak JE, Ross-Macdonald PB, Roeder GS (2001) The budding yeast Msh4 protein functions in chromosome synapsis and the regulation of crossover distribution. Genetics 158: 1013-1025.

31. Ross-Macdonald P, Roeder GS (1994) Mutation of a meiosis-specific MutS homolog decreases crossing over but not mismatch correction. Cell 79: 1069-1080.

32. Rockmill B, Fung JC, Branda SS, Roeder GS (2003) The Sgs1 helicase regulates chromosome synapsis and meiotic crossing over. Curr Biol 13: 1954-1962.

33. Henderson KA, Keeney S (2004) Tying synaptonemal complex initiation to the formation and programmed repair of DNA double-strand breaks. Proc Natl Acad Sci U S A 101: 4519-4524.

34. Marcon E, Moens P (2003) MLH1p and MLH3p localize to precociously induced chiasmata of okadaic-acid-treated mouse spermatocytes. Genetics 165: 2283-2287.

35. Watt PM, Hickson ID, Borts RH, Louis EJ (1996) SGS1, a homologue of the Bloom's and Werner's syndrome genes, is required for maintenance of genome stability in Saccharomyces cerevisiae. Genetics 144: 935-945.

36. Spell RM, Jinks-Robertson S (2004) Examination of the roles of Sgs 1 and Srs2 helicases in the enforcement of recombination fidelity in Saccharomyces cerevisiae. Genetics 168: 1855-1865.

37. Sugawara N, Goldfarb T, Studamire B, Alani E, Haber JE (2004) Heteroduplex rejection during single-strand annealing requires Sgs1 helicase and mismatch repair proteins Msh2 and Msh6 but not Pms1. Proc Natl Acad Sci U S A 101: 9315-9320.

38. Myung K, Datta A, Chen C, Kolodner RD (2001) SGS1, the Saccharomyces cerevisiae homologue of BLM and WRN, suppresses genome instability and homologous recombination. Nat Genet 27: 113-116.

39. Chaganti RS, Schonberg S, German J (1974) A manyfold increase in sister chromatid exchanges in Bloom's syndrome lymphocytes. Proc Natl Acad Sci U S A 71: 4508-4512.

40. Wang W, Seki M, Narita Y, Sonoda E, Takeda S, et al. (2000) Possible association of BLM in decreasing DNA double strand breaks during DNA replication. EMBO J 19: 3428-3435.

41. Mankouri HW, Hickson ID (2004) Understanding the roles of RecQ helicases in the maintenance of genome integrity and suppression of tumorigenesis. Biochem Soc Trans 32: 957-958.

42. Wu L, Hickson ID (2003) The Bloom's syndrome helicase suppresses crossing over during homologous recombination. Nature 426: 870-874.

43. Bennett RJ, Sharp JA, Wang JC (1998) Purification and characterization of the Sgs 1 DNA helicase activity of Saccharomyces cerevisiae. J Biol Chem 273: 9644-9650.

44. Ira G, Malkova A, Liberi G, Foiani M, Haber JE (2003) Srs2 and Sgs1-Top3 suppress crossovers during double-strand break repair in yeast. Cell 115: 401-411.

45. Robert T, Dervins D, Fabre F, Gangloff S (2006) Mrc1 and Srs2 are major actors in the regulation of spontaneous crossover. EMBO J 25: 2837-2846.

46. Mullen JR, Kaliraman V, Brill SJ (2000) Bipartite structure of the SGS1 DNA helicase in Saccharomyces cerevisiae. Genetics 154: 1101-1114.

47. Nakagawa T, Ogawa H (1999) The Saccharomyces cerevisiae MER3 gene, encoding a novel helicase-like protein, is required for crossover control in meiosis. EMBO J 18: 5714-5723.

48. Sym M, Roeder GS (1995) Zip1-induced changes in synaptonemal complex structure and polycomplex assembly. J Cell Biol 128: 455-466.

49. Smith AV, Roeder GS (1997) The yeast Red1 protein localizes to the cores of meiotic chromosomes. J Cell Biol 136: 957-967. 
50. Zickler D, Kleckner N (1998) The leptotene-zygotene transition of meiosis Annu Rev Genet 32: 619-697.

51. Argueso JL, Wanat J, Gemici Z, Alani E (2004) Competing crossover pathways act during meiosis in Saccharomyces cerevisiae. Genetics 168: 18051816.

52. de los Santos T, Hunter N, Lee C, Larkin B, Loidl J, et al. (2003) The Mus81/ Mms4 endonuclease acts independently of double-Holliday junction resolution to promote a distinct subset of crossovers during meiosis in budding yeast. Genetics 164: 81-94.

53. Rockmill B, Sym M, Scherthan H, Roeder GS (1995) Roles for two RecA homologs in promoting meiotic chromosome synapsis. Genes Dev 9: 26842695.

54. Kane SM, Roth R (1974) Carbohydrate metabolism during ascospore development in yeast. J Bacteriol 118: 8-14.

55. Wach A, Brachat A, Pohlmann R, Philippsen P (1994) New heterologous modules for classical or PCR-based gene disruptions in Saccharomyces cerevisiae. Yeast 10: 1793-1808.

56. Lee BH, Amon A (2003) Role of Polo-like kinase CDC5 in programming meiosis I chromosome segregation. Science 300: 482-486. 